\title{
Study of Water Permeability of Lightweight Concrete
}

\author{
Xiaoxiao Xu, Haibin Yang, Hongzhi Cui, and Zitao Lin \\ Guangdong Provincial Key Laboratory of Durability for Marine Engineering, College of Civil Engineering, \\ Shenzhen University, Shenzhen 518060, China \\ Tommy Yiu Lo \\ Department of Engineering and Architecture, City University of Hong Kong
}

\begin{abstract}
The relationship between water permeability and different levels of the tested layers from the top surface to the bottom surface of the lightweight concrete specimens is determined in this study. It is beneficial to develop the design criteria for a durable lightweight concrete. The water permeability coefficient $\mathrm{K}$ of the samples was determined by water permeability test using GWT. It is found that the water permeability coefficient K of the three layers in each set of samples tends to decrease as the level of the tested from the top surface increase. Larger Rebound Number and higher density in the bottom layer and more coarse lightweight aggregates in top layer correlate with the result of the descending water permeability trend and also indicate the existence of floatation of lightweight aggregates. Therefore, concrete uniform is very important for lightweight concrete.
\end{abstract}

Keywords: lightweight concrete, water permeability, durability, strength

\section{INTRODUCTION}

It is every construction person's dream that the construction can maintain its using function for a long time and with a lower cost to keep a good repair. However, the durability of concrete, in fact, is definite. Deterioration of concrete is a common phenomenon. One of the causes of deterioration is by chemical attacks, such as carbonation and corrosion of steel reinforcement. Since the aggressive chemical ions, such as chlorides, sulphates, carbon dioxide, and even sea water, arise externally from environment and the attack takes place within the concrete mass, so the adverse chemical external must have the way to penetrate into the concrete structure. Thus, it can be trusted that the concrete must be permeable. The property of the permeability in concrete decides the quantity, methods, depth, and velocity of the chemical attacks. Permeability is, therefore, of critical interest, especially in concrete used for water training structures or watertight basement.

Recently, considerable attention has been paid to permeability of concrete. Due to the limited general knowledge about techniques for measuring permeability, no standard procedure is available for this approach. A wide range of testing techniques and fundamental theory is reviewed comprehensively in Concrete Society Technical Report 31 (1988). Owing to the most of the tests are carried out on-site in insite concrete, a limitation of obtaining results only in surface zone occurs. However, the permeability of lightweight concrete seems to be neglected.
With the repaid usage of the lightweight concrete all around the world, the survey on the permeability of lightweight concrete is worth to be carried out. It is inconvenient that test on the surface zone of in-site concrete to present the permeability of the lightweight concrete due to the floatation of lightweight aggregates. In order to better measure the permeability of the lightweight concrete, particular attention is required in determining the relationship between permeability and the levels of the tested layer from the surface.

The present research aims to determine the relationship between water permeability and levels of tested layer from the top surface of laboratory lightweight concrete specimens and to develop design criteria for a durable lightweight concrete. Referring to the literature review, the GWT test and Rebound Hammer test were mainly used in this research.

\section{THE EXPERIMENTAL PROGRAM}

\subsection{Water permeability test by GWT (GWT manual)}

GWT is used to evaluate the surface porosity, such as microcracking and air bubbles, and to test waterproofing membrane. If the concrete surface without porosity and if the concrete is saturate with concrete, GWT is a wonderful measurement of the permeability without the disturbance by capillary absorption caused by testing a dried out concrete.

In this test, a pressure housing with build-in micrometer adjustable gauge, pressure meter 0-6 bar, pressure lid with O-ring, key for turning lid, valves, and watertight 
gasket, water filling cup attached to L-joint, and two adjustable clamping pliers are needed. The pressure housing is clamped to the concrete surface by two pliers. The housing is sealed by means of a gasket being compressed. After the housing is filled with water, valves are closed, and pressure is established by turning the adjustable nut on the housing. This reduces the volume. Water is squeezed through the concrete under pressure. The pressure inside the housing then begins to fall since some of the micrometer screw gauge to reduce the volume for a certain time. The reading of the micrometer screw gauge before and after the test gives a measure of the volume of water squeezed into the concrete at this pressure. These reading and the reading of time are used to calculate the coefficient of water permeability. The cross-section of the apparatus is shown in Figure 13.

The flux q of water into concrete is then calculated by the following equation:

$$
q=78.6 \times(g 2-g 1) / 3018 \times \text { time }
$$

q: flux of water into concrete

g1: reading before the test

g2: reading after the test

78.6: an area of the micrometer gauge into the pressure housing $\left(\mathrm{mm}^{2}\right)$

3018: an area of the pressure surface towards the concrete $\left(\mathrm{mm}^{2}\right)$.

The coefficient of water permeability is then calculated by the following equation:

$$
\mathrm{q}=(\mathrm{b} \times \mathrm{k} \times \Delta \mathrm{p}) / \mathrm{L}
$$

$b$ : the percentage of cement paste in the concrete

$k$ : the coefficient of water permeability

$\Delta \mathrm{p}$ : pressure of the meter

$\mathrm{L}$ : thickness of the concrete

\subsection{Surface hardness test by rebound hammer (BS 1881: Part: 202: 1996)}

Rebound hammer test is a non-destructive method of testing the hardness of concrete surface. The test is based on the principle that the rebound of an elastic mass with a standardized energy depends on the hardness of the surface when it impinges. The amount of the measurement is expressed as a Rebound Number. It is a measure of a relative hardness of the surface of the concrete in which thickness no more than $30 \mathrm{~mm}$. Rebound hummer is a test to check the uniformity of the concrete and make an approximate evaluation of the strength of concrete.

\subsection{Materials and apparatus}

(1) Lightweight Aggregate-Leca with size $<6 \mathrm{~mm}$ (see Figure 1)

(2) Portland cement

(3) River sand
(4) Water

(5) Water Reducing Admixture-WRDA88 (see Figure 2)

(6) Superplasticizer-Daracem 100 (see Figure 3)

(7) Oven (see Figure 4)

(8) Weighing Balance

(9) $3 \mathrm{dm}^{3}$ container

(10) Sieves and shaker

(11) $100 \mathrm{~mm} \times 200 \mathrm{~mm}$ Cylinder Mould and $100 \mathrm{~mm} \times 100 \mathrm{~mm} \times 100 \mathrm{~mm}$ Cube Mould (see Figure 5)

(12) Slump Cone

(13) Concrete Mixer

(14) $27^{\circ} \mathrm{C}$ water tank (see Figure 6)

(15) Compression machine (see Figure 7)

(16) Concrete cutting machine (see Figure 8)

(17) Grinder (see Figure 9)

(18) Vacuum pump (see Figure 10)

(19) GWT (see Figure 11)

(20) Rebound Hammer-Type L (see Figure 12)

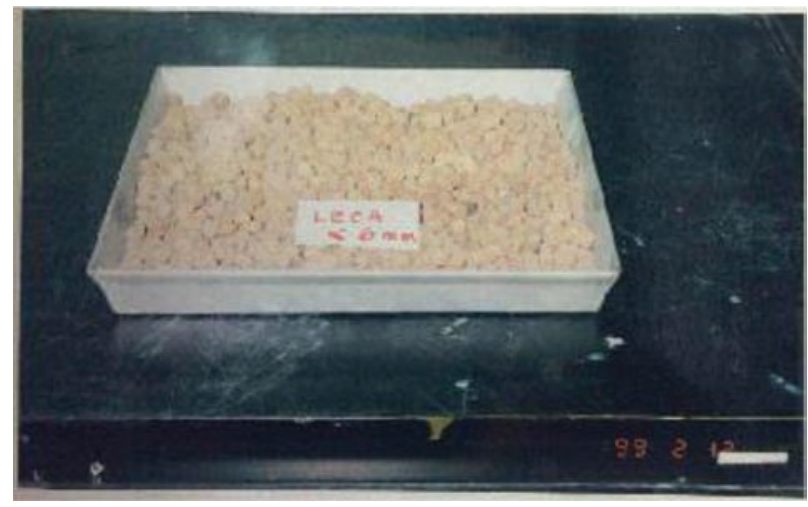

Figure 1. Lightweight aggregate-leca with size $<6 \mathrm{~mm}$.

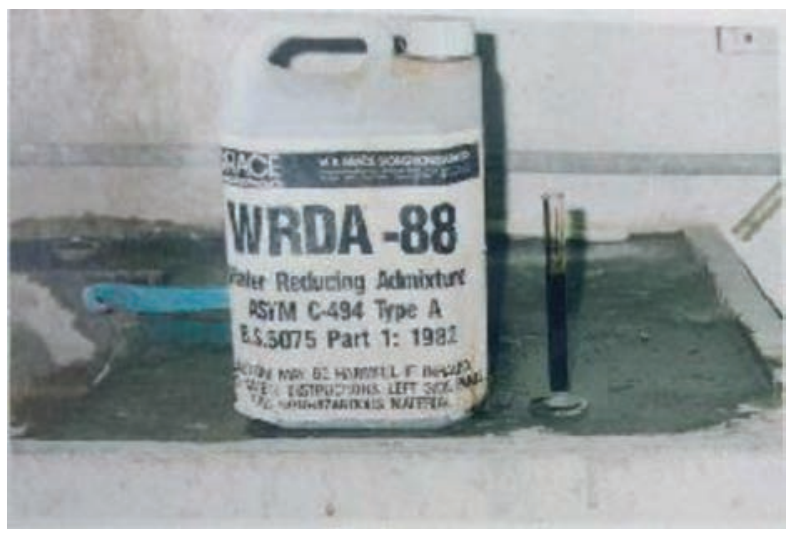

Figure 2. Water reducing admixture-WRDA88. 


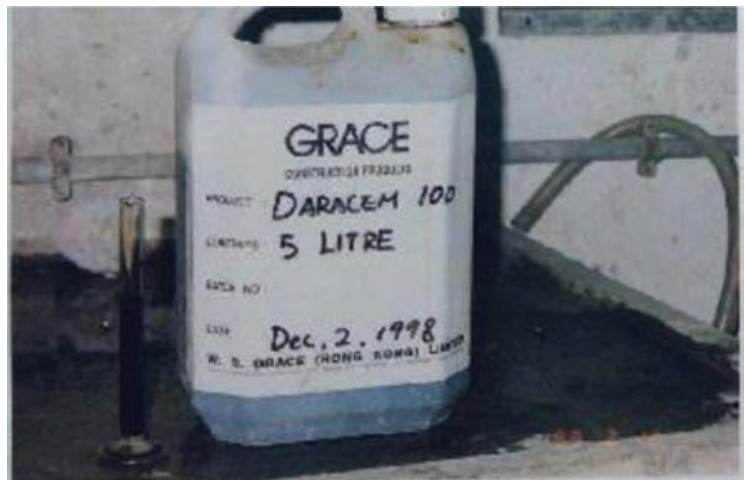

Figure 3. Superplasticizer-Daracem 100.

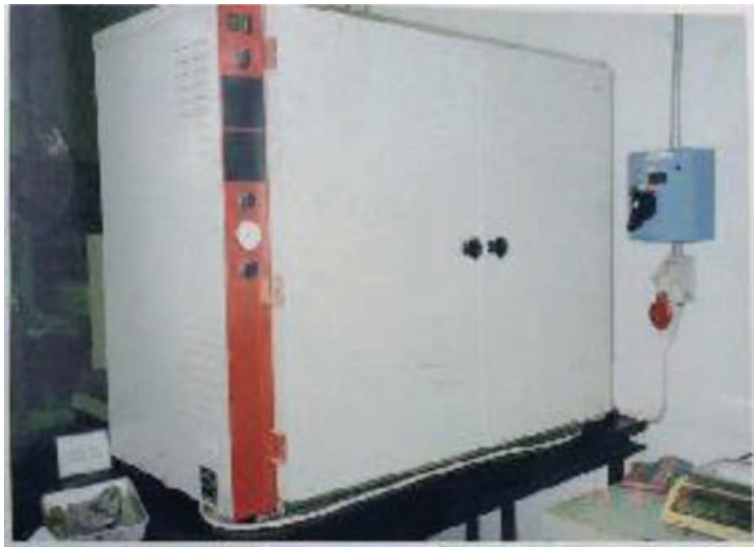

Figure 4. Oven.

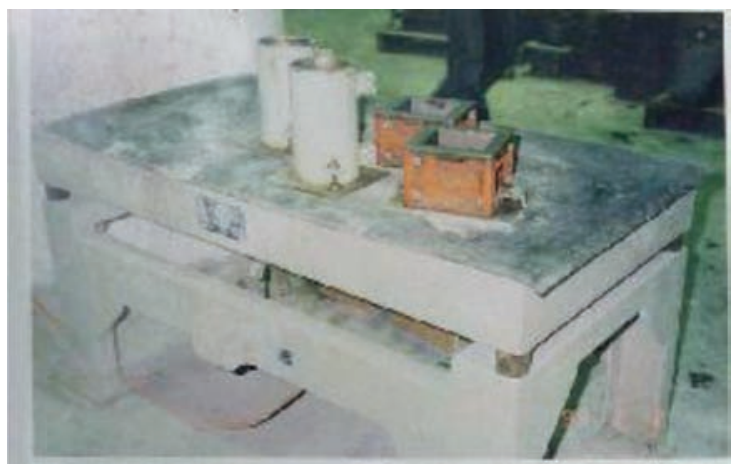

Figure $5.100 \mathrm{~mm} \times 200 \mathrm{~mm}$ cylinder mould and $100 \mathrm{~mm} \times 100 \mathrm{~mm} \times$ $100 \mathrm{~mm}$ cube mould.

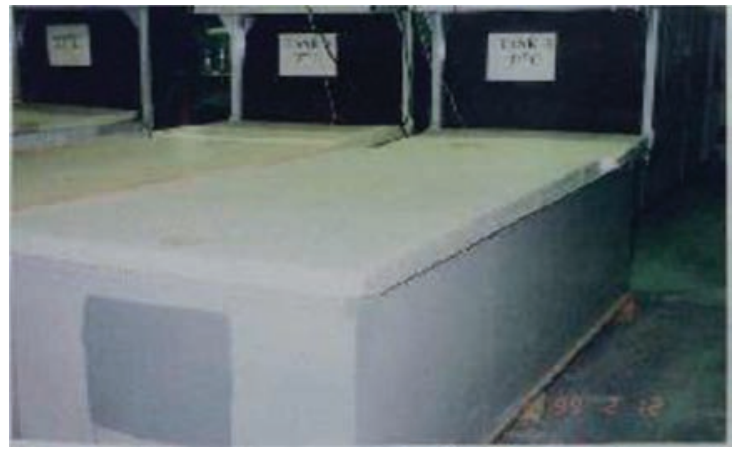

Figure 6. Water tank.

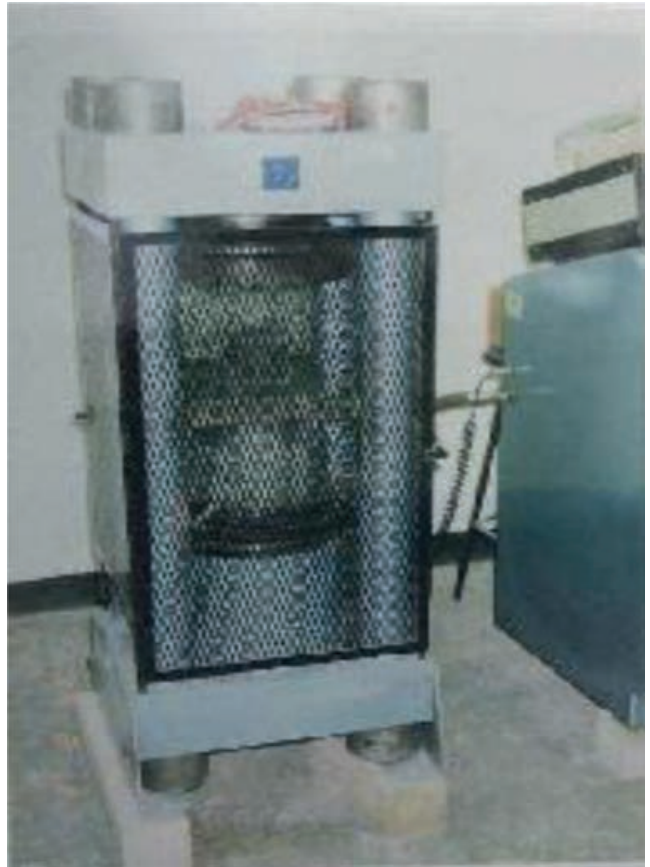

Figure 7. Compressive machine.

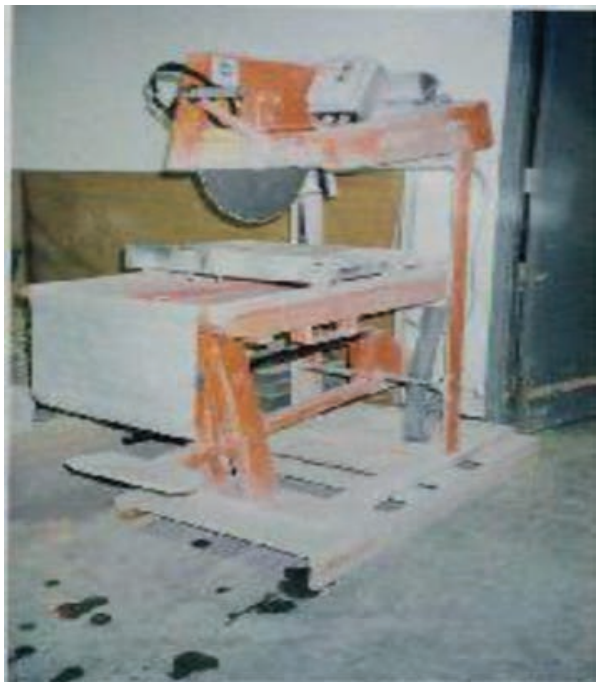

Figure 8. Concrete cutting machine.

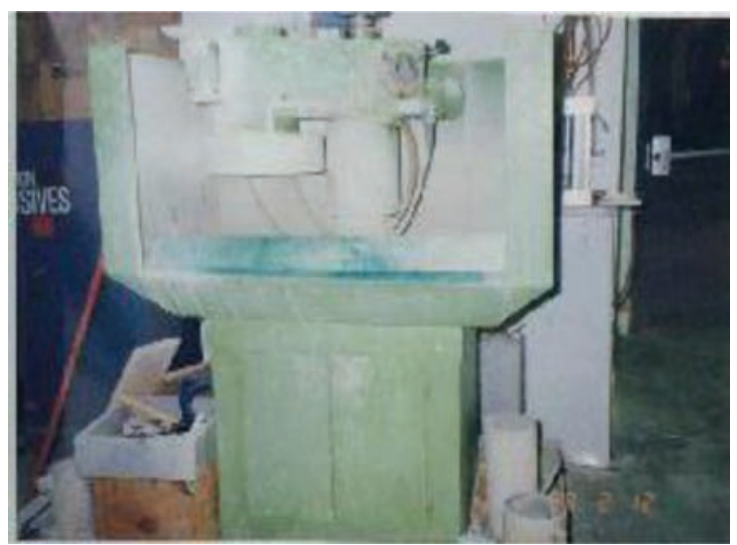

Figure 9. Grinder. 


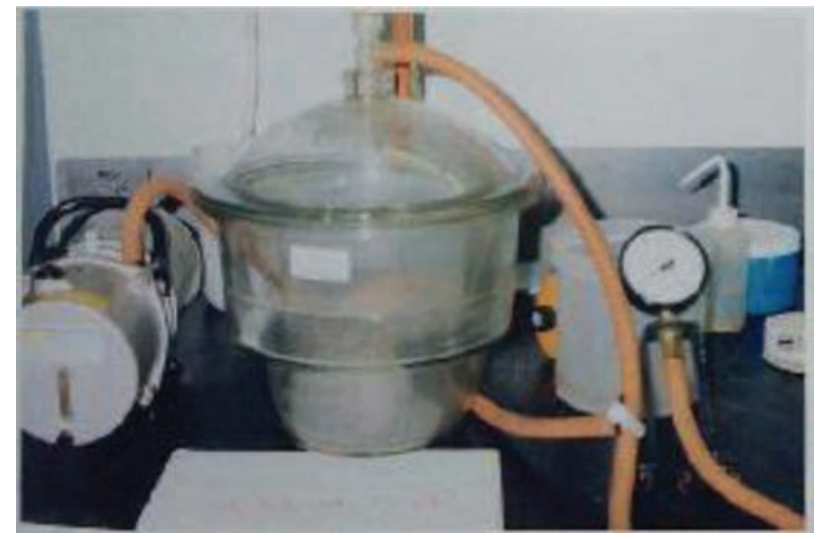

Figure 10. Vacuum pump.

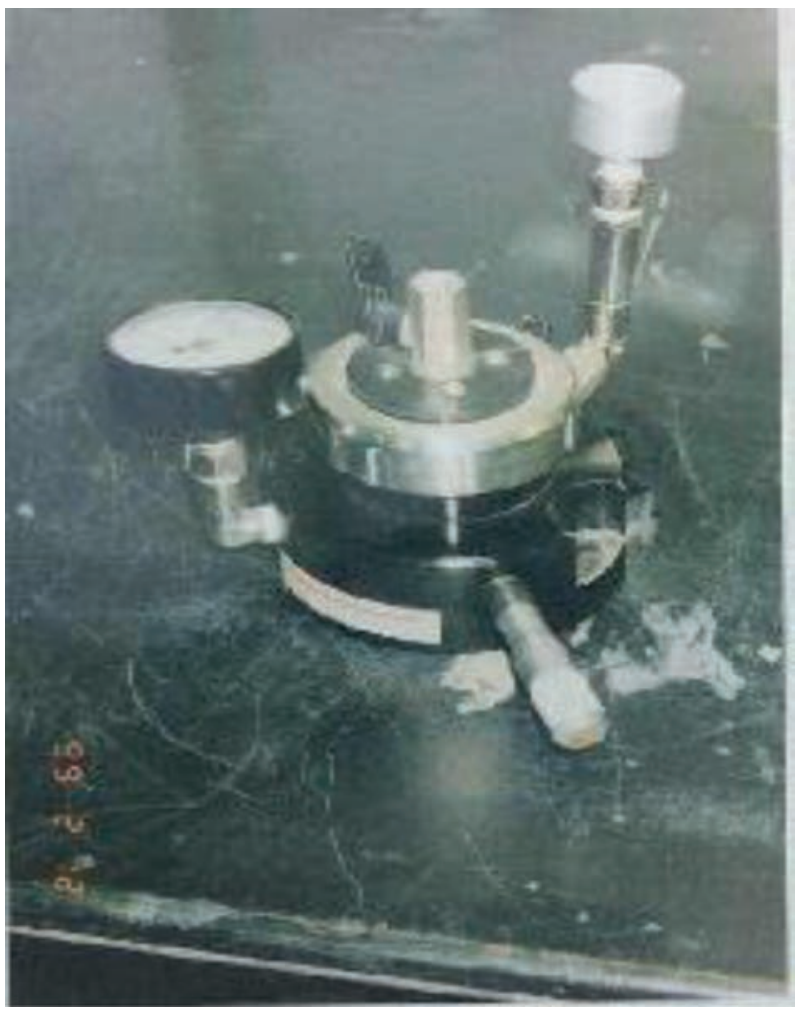

Figure 11. GWT.

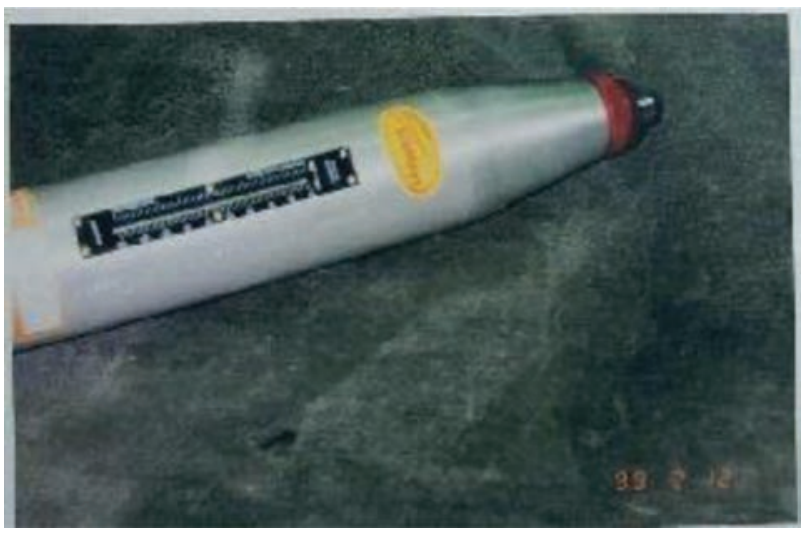

Figure 12. Rebound Hammer-Type L.

\subsection{Methods}

2.4.1 Method for determination of loose bulk density (BS 3797: 1990)

All aggregates samples were dried by an oven first. The $3-\mathrm{dm}^{3}$ container was calibrated by a balance, and then the container was placed on a horizontal surface and filled to overflowing by discharging the aggregates from a scoop, from a height not exceeding $50 \mathrm{~mm}$ above the top of the container. The surface of the aggregates was leveled using the straightedge. The container was then weighted, and the loose bulk density of the aggregate was then determined. The above steps were repeated by four times more. The results of loose bulk density are shown in Table 1.

Table 1. Result of loose bulk density.

\begin{tabular}{lccccc}
\hline Test cube & $\mathbf{1}$ & $\mathbf{2}$ & $\mathbf{3}$ & $\mathbf{4}$ & $\mathbf{5}$ \\
\hline Weight of & 1836.2 & 1813.7 & 1803.5 & 1821.8 & 1817.8 \\
aggregate $(\mathrm{g})$ & & & & & \\
Average $(\mathrm{g})$ & & & 1818.6 & & \\
Loose bulk & & & 606.2 & & \\
density $\left(\mathrm{kg} / \mathrm{m}^{3}\right)$ & & & & & \\
\hline
\end{tabular}

\subsubsection{Method for determination of aggregates size distribution (BS812:Part 103: 1985)}

First, all aggregate samples were dried by an oven, and then evenly divided by a riffle box. The test aggregates were weighted by a balance, and then were placed on the top coarsest sieve and covered with a lid. The nest of the sieves was shaken by a mechanical shaker for at least 2 min to separate the aggregates into the size fraction of the sieves. At least, the aggregates retained on each sieve were weighted after the completion of sieving. The results of aggregate size distribution are shown in Table 2, and the grading curve is shown in Figure 13.

Table 2. Result of aggregate size distribution.

\begin{tabular}{|c|c|c|c|}
\hline \multicolumn{4}{|c|}{ Total weight of sample $(\mathrm{g}): 105.1$} \\
\hline \multirow{2}{*}{$\begin{array}{l}\text { B.S.S. } \\
\text { Sieve }(\mathrm{mm})\end{array}$} & \multicolumn{2}{|c|}{ Individual retained } & \multirow{2}{*}{$\begin{array}{c}\text { Accumulate passing } \\
(\%)\end{array}$} \\
\hline & (g) & $(\%)$ & \\
\hline 5.000 & 30.1 & 28.6 & 71.4 \\
\hline 2.360 & 54.2 & 51.6 & 19.8 \\
\hline 1.180 & 14.8 & 14.1 & 5.7 \\
\hline 0.600 & 2.3 & 2.2 & 3.5 \\
\hline 0.300 & 1.2 & 1.1 & 2.4 \\
\hline 0.150 & 0.9 & 0.9 & 1.5 \\
\hline 0.075 & 0.4 & 0.4 & 1.1 \\
\hline \multirow[t]{2}{*}{ Pan } & 0.3 & 0.3 & \\
\hline & al: 104.2 & & \\
\hline
\end{tabular}




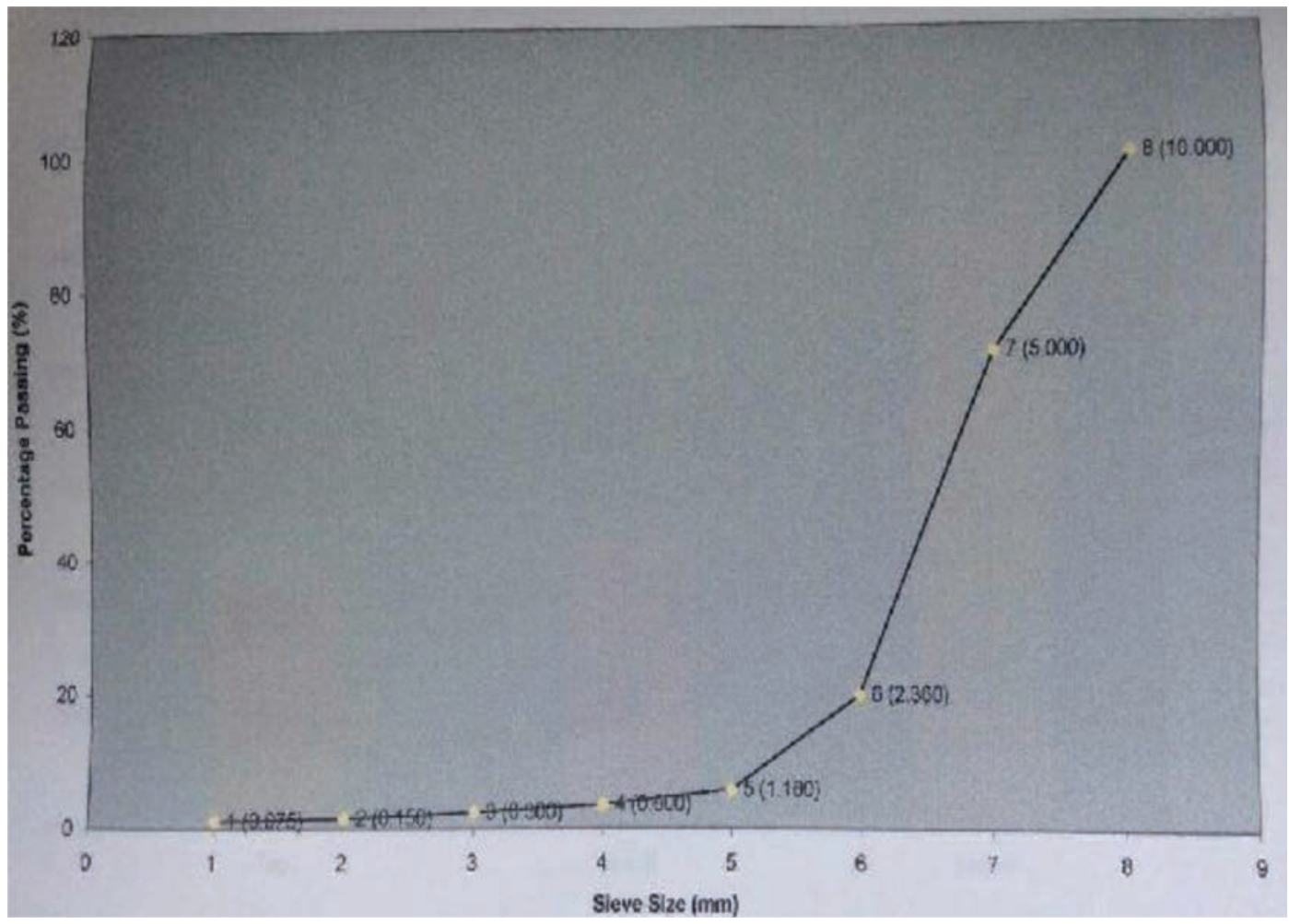

Figure 13. Grading curve of sieve analysis.

\subsubsection{Design mix proportion}

The design mix proportion is shown in Table 3.

Table 3. Design mix proportion.

\begin{tabular}{ccccc}
\hline \multicolumn{5}{c}{ Superplasticizer: $\mathbf{6 5} \mathbf{~ m l}$} \\
\hline $\begin{array}{c}\text { Slump: } 55 \mathbf{~ m m} \\
\text { Portland } \\
\text { cement }(\mathbf{k g})\end{array}$ & $\begin{array}{c}\text { Leca } \\
<\mathbf{6 m}(\mathbf{k g})\end{array}$ & $\begin{array}{c}\text { River } \\
\text { sand }(\mathbf{k g})\end{array}$ & $\begin{array}{c}\text { Water } \\
(\mathbf{k g})\end{array}$ & WRDA (ml) \\
\hline 10.35 & 11.82 & 17.09 & 5.82 & 51.75 \\
\hline
\end{tabular}

\subsubsection{Method for making test cubes, specimens, and} curing (BS 1881: Part 110\&111: 1983)

Eight cylinder moulds and five cube moulds were used to make specimens and test cubes. The moulds have brushed with oil, which were placed on a vibrating table, and then the fresh concrete was placed into the moulds by scoop in three layers. Each layer was vibrated until the surface was relatively smooth to guarantee the compaction of the concrete. The top layer was smoothed to level with the top of the mould by a float after compaction. Each specimen was covered with the polythene sheets to preserve the water and for curing $24 \mathrm{~h}$. The specimens were removed from the moulds after $24 \mathrm{~h}$ and marked for identification and stored in the curing tank for 28 days. Three layers named top, middle, and bottom, respectively, which thickness is $25 \mathrm{~mm}$ cut from each cylinder by the concrete cutting machine after the completion of curing and each surface of the each layer was smoothed by the grinder (Figure 14).

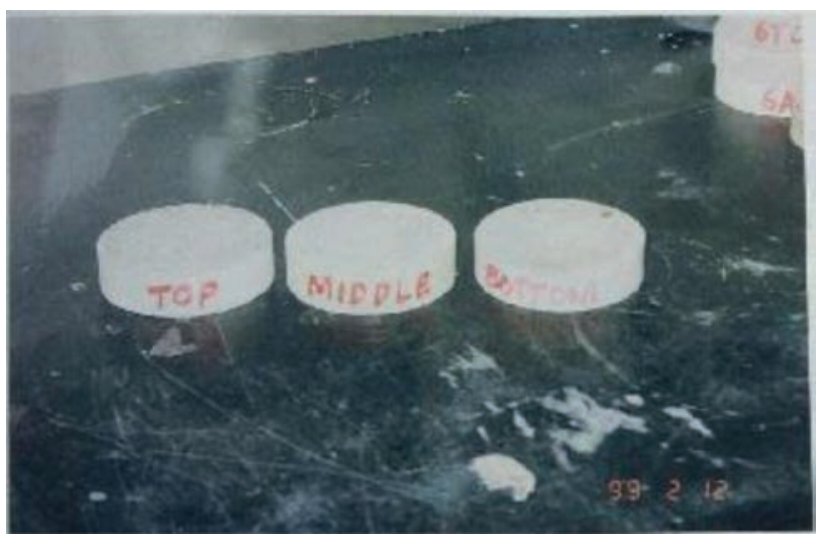

Figure 14. A set of sample.

\subsubsection{Determination of compression strength of concrete cube}

In this experiment, five cubes, which dimension is $100 \mathrm{~mm} \times 100 \mathrm{~mm} \times 100 \mathrm{~mm}$, will be placed on the surface of the compression machine and the compression strength was then calculated by dividing the maximum load by the cross-sectional area of the cube.

\subsubsection{Determination of sample density by water displacement}

Eight sets of sample with top, middle, and bottom layers each were first saturated by vacuum pump for $4 \mathrm{~h}$. The surface of the sample was dried by cloth after saturation. The sample was weighted by a balance, 
and the reading was recorded. The sample was placed on the hanger immersed in the water tank under the balance and weighted. It is easy to know that the difference between the two readings was the mass of water displaced when the sample was immersed into the water.

\subsubsection{Method for determination of water permeability by GWT (GWT manual)}

The samples were first saturated by vacuum pump for $4 \mathrm{~h}$. Two clamping pliers were tightly secured to two concrete between the two concrete blocks separately. The test specimen was placed on a wooden plate located between the two blocks. The housing was placed on the test specimen and fixed in position tightly by the two pliers (Figure 15). The water tight gasket was fully compressed and invisible. The values were opened, and the cup was filled with water until water was expelled from the other valve to ensure the chamber was totally filled with water. The micrometer gauge was turned to $0.00 \mathrm{~mm}$ as the starting point. The valve was closed and pressure was applied by turning the micrometer gauge, and the stop watch was started simultaneously. The micrometer gauge was stopped to turn once as the stop watch was stopped, then the stop watch reading and micrometer gauge reading were recorded. Those steps were repeated for other samples.

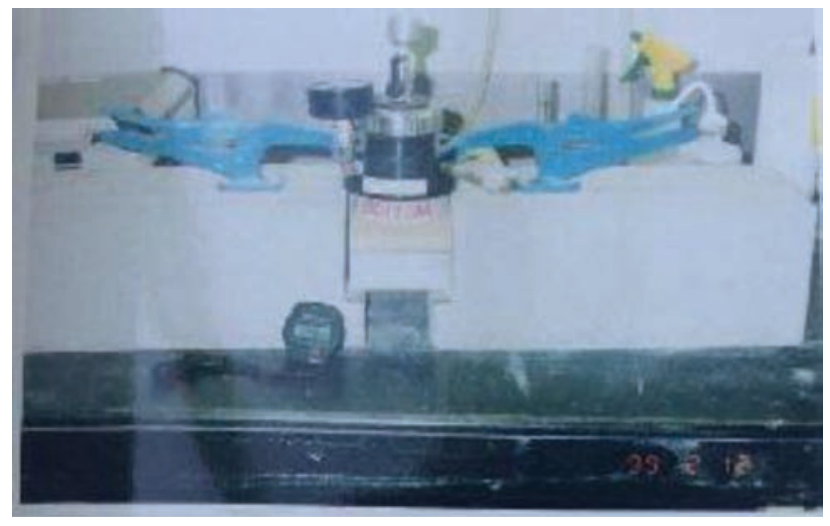

Figure 15. A setup of water permeability test.

\subsubsection{Determination of the surface aggregate distribution and the surface hardness}

In order to determinate the surface aggregate distribution, the surface of the sample was wet and cleaned by a brush, and then a transparent graph paper with $5 \mathrm{~mm} / \mathrm{U}$ was placed on the sample. The surface aggregate with size larger than $5 \mathrm{~mm}$ was marked in red color (Figure 16). The above steps were repeated for other test samples.

Rebound Hammer was used to determine the surface hardness. Type $L$ hammer whose impact energy was $0.735 \mathrm{Nm}$ was used for the test. At first, the test points of a dry and clean sample were selected, then the sample was placed on a smooth and ridge surface like a heavy plate (Figure 17). The edges of the sample were stepped by foot to prevent the movement of the samples. Ten points selected at first on the area of the sample were rebounded, and the readings were recorded. As calculating the mean value from the mean by more than $5 \mathrm{U}$ was eliminated, and this hammer reading was placed by a further impact test.

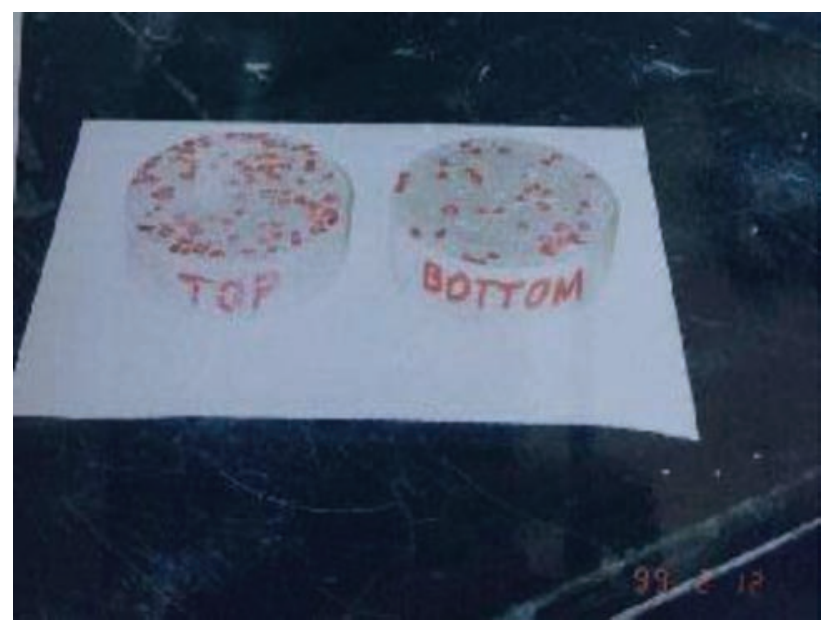

Figure 16. Marked surface aggregate with size larger than $5 \mathrm{~mm}$.

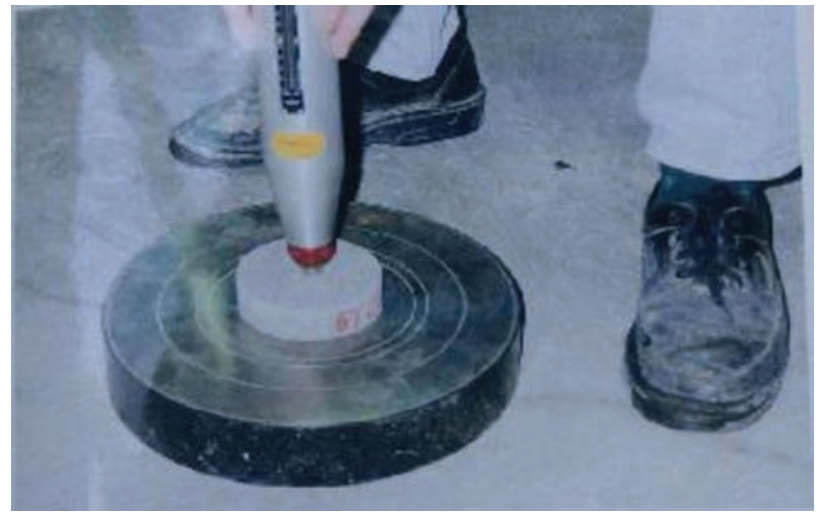

Figure 17. A setup of Rebound Hammer test.

\section{RESULTS AND DISCUSSION}

\subsection{The result of compression test is shown in Table 4}

Table 4. Result of compression test.

\begin{tabular}{lccccc}
\hline Test cube & $\mathbf{1}$ & $\mathbf{2}$ & $\mathbf{3}$ & $\mathbf{4}$ & $\mathbf{5}$ \\
\hline Failure load (KN) & 370.1 & 337.8 & 362.2 & 311.3 & 350.1 \\
Average (KN) & & & 346.3 & & \\
Compression & & & 34.6 & & \\
strength (N) & & & & & \\
\hline
\end{tabular}




\subsection{The results of sample density by water displacement method}

The eight sets of result of sample density in different layers by water displacement method are shown in Table 5. According to the eight sets of result, the sample densities of the top layers have a range from 1640.87 to $1670.58 \mathrm{~kg} / \mathrm{m}^{3}$. The sample densities of the middle layers have a range from 1655.37 to $1695.92 \mathrm{~kg} / \mathrm{m}^{3}$, and the sample densities of the bottom layers have a range from 1840.25 to $2019.97 \mathrm{~kg} / \mathrm{m}^{3}$. The sample density has an increasing trend as the level of the tested layer increases. Moreover, the density of the bottom layer is particularly high when compared with the top and middle layers. The top layer of the sample has the lowest density, because there are most coarse aggregates in this portion as discussed in previous section. Since the coarse aggregate is quite lightweight, they own high porosity and the air voids within these coarse aggregate lead to materials float on the top surface. In consequence, the large quantity of air voids within the volume of the hardened top layer causes the lowest density. So, the number of the coarse aggregate is, in middle and bottom layer, reduced gradually and correspond to the decrease of the volume of the coarse aggregate in the bottom layer, the density of the bottom layer increased. The reason to explain the high density of the bottom layer is that the higher specific gravity river sand or cement paste is settled at the bottom of the mould during mixing due to vibration of the fresh concrete.

\subsection{Results of water permeability test by GWT}

The eight sets of result of water permeability in different layers are shown in Table 6.

According to the results of the GWT test on eight sets, the water permeability coefficient $\mathrm{K}$ of the top layers $(5-30 \mathrm{~mm})$ has a range from $3.39 \times 10^{-9}$ to $3.65 \times 10^{-9} \mathrm{~m} / \mathrm{s}$. The water coefficient $\mathrm{K}$ of the middle layers $(90-115 \mathrm{~mm})$ has a range from $2.83 \times 10^{-9}$ to
$3.18 \times 10^{-9} \mathrm{~m} / \mathrm{s}$, and the water permeability coefficient $\mathrm{K}$ of the bottom layers $(175-200 \mathrm{~mm})$ has a range from $1.41 \times 10^{-9}$ to $1.69 \times 10^{-9} \mathrm{~m} / \mathrm{s}$. The water permeability has an increasing trend from the bottom layer to the top layer.

The top layer of the sample has the highest water permeability coefficient $\mathrm{K}$. According to the results of surface aggregate distribution, the top layer contains the most air-filled coarse aggregate of mix. It can be explained that the density of the top layer is the lowest due to the large air voids within the coarse aggregates. As the packing of the coarse aggregates is poor due to their large size and bulk shape, the size of the voids between them is large with consequence of low bound strength between coarse aggregates by the matrix. Therefore, the strength of the top layer is low. Meantime, the permeability of the top layer is high. Larger voids between the aggregates means more cement paste filled. The cement paste content between the aggregates is a crucial factor when considering the permeability of different layers. More cement paste filled the voids between the aggregates in the top layer means more permeable of the concrete. The weak bond between the coarse aggregates and the matrix also offers an easier flow path around the aggregates and the increase the permeability of the top layer. Furthermore, owing to the weak strength of the top layer, the risk of microcracks caused by the shrinkage of the matrix is higher in the top layer than in the middle or in bottom layer.

According to the results of surface size distribution, the number of coarse aggregates is reduced in the middle layer and bottom layer with the density of each layer increased gradually. Decreasing size of the coarse aggregates, the size of the voids reduces correspondingly due to the improvement in packing of the aggregates in the middle and bottom layer. Thus, the bond strength between the aggregates and the matrix increases and the strength of the hardened

Table 5. Relation between density and different layers of eight samples.

\begin{tabular}{|c|c|c|c|c|c|c|c|c|}
\hline Test sample & 1 & 2 & 3 & 4 & 5 & 6 & 7 & 8 \\
\hline Top density $\left(\mathrm{kg} / \mathrm{m}^{3}\right)$ & 1659.07 & 1659.89 & 1665.66 & 1657.63 & 1640.87 & 1645.03 & 1654.41 & 1670.58 \\
\hline Middle density $\left(\mathrm{kg} / \mathrm{m}^{3}\right)$ & 1682.61 & 1672.59 & 1694.09 & 1682.13 & 1655.37 & 1685.91 & 1655.91 & 1695.92 \\
\hline Bottom density $\left(\mathrm{kg} / \mathrm{m}^{3}\right)$ & 1840.25 & 1888.55 & 1901.72 & 1993.92 & 1908.03 & 2019.97 & 1972.59 & 1948.06 \\
\hline
\end{tabular}

Table 6. Relation between water permeability and different layers of eight samples and $\mathrm{K}$ is permeability coefficient $\left(10^{-9} \mathrm{~m} / \mathrm{s}\right)$.

\begin{tabular}{lcccccccc}
\hline Distance to top layer & Sample 1 & Sample 2 & Sample 3 & Sample 4 & Sample 5 & Sample 6 & Sample 7 & Sample 8 \\
\hline K of $30 \mathrm{~mm}$ & 3.52 & 3.49 & 3.41 & 3.53 & 3.65 & 3.61 & 3.62 & 3.39 \\
K of $115 \mathrm{~mm}$ & 2.92 & 2.99 & 2.97 & 2.88 & 3.11 & 2.83 & 3.18 \\
K of $200 \mathrm{~mm}$ & 1.56 & 1.63 & 1.69 & 1.41 & 1.59 & 1.49 & 1.53 & 1.43 \\
\hline
\end{tabular}


cement paste is then strengthened, which is based on the results of surface hardness test by Rebound Hammer. However, the decreasing permeability of the middle and bottom layer owing to the reason that decreasing in size of voids between aggregates enables less cement paste filled with a consequence of less permeable of concrete and make the concrete more fine and close. The fine sand improves the packing of aggregates, so that the size of voids between the aggregates in the bottom layer is the smallest, which better explain the highest strength and bond strength of the bottom layer.

\subsection{Surface hardness test by Rebound Hammer}

Since the Rebound Number is directly proportional to the cube compressive strength in accordance with the calibration curve, the comparison of the Rebound Number is representative enough to indicate the surface hardness. According to the eight sets of the result shown in Table 7, the Rebound Numbers in the top layer have a range from 23.6 to 25.9. The Rebound Numbers in the middle layer have a range from 24.4 to 27.9, and the Rebound Numbers in the bottom layers have a range from 26.3 to 30.1 . Similar to the results of sample density, the Rebound Number has an increasing trend as the level of the tested layer increases. Moreover, the Rebound Number in the bottom layer is particularly large when compared with the top and middle layers. Strength of concrete is governed by cement paste and aggregate characteristics, so the influence of aggregate type and proportions is considerable. The Rebound Number in the top layer is the smallest, and the top layer has the lowest strength. Since the most lower density coarse aggregates float on the top layer, the total exposed coarse aggregates on the surface of the top layer is larger than the middle and bottom layer. For hardened paste, since the size of the coarse aggregates are also large because of poor packing. The matrix network around the aggregates is therefore weakened. As a result, the strength of the hardened paste is low. As the number of coarse aggregates is reduced in the middle layer and bottom layer in accordance with the results from surface aggregates distribution, the area of cellular structure of the coarse aggregates exposed to the section surface is also reduced relatively. The influence to the concrete strength due to the cellular structure of aggregate is reduced, thus increasing the strength. Besides, since the maximum particle diameter in the middle layer and bottom layer is gradually lowered, the packing of the aggregates is better, and therefore the size of the voids between the aggregates is reduced. As a result, the matrix bond between cement paste and aggregates is strengthened. Moreover, the Rebound Number in the bottom layer is particularly large. Most of the natural fine sand is settled on the bottom layer by vibration and cause high density of sample. The packing of the aggregates at the bottom layer is improved not only due to reduction in size of voids between the aggregates but also due to sufficient addition of fine sand between them. Since fine sand can improve workability and increase strength of hardened paste by strengthen the matrix network around aggregates, the strength in the bottom layer as a whole is particularly increased.

\subsection{Surface aggregates distribution}

According to the eight sets of the result shown in Table 8, the number of aggregates with size larger than $5 \mathrm{~mm}$ on the surface of the top layers has a range from 69 to 79 . The number of aggregates with size larger than $5 \mathrm{~mm}$ on the surface of the middle layers has a range from 37 to 41 , and the number of aggregates with size larger than $5 \mathrm{~mm}$ on the surface of the bottom layers has a range from 25 to 31 . Leca, which is the lightweight aggregate used in this experiment, with size $<6 \mathrm{~mm}$ and more than $5 \mathrm{~mm}$ is particularly more on the surface of the top layer section than those on the surface of the middle and bottom layer section. The phenomenon is owing to the tendency of coarse lightweight aggregates to float onto the top surface of $100 \times 200$ cylindrical moulds. This phenomenon is known as segregation. In the case of concrete, the primary causes of segregate are the differences in the size of particles and in the specific gravity of the mix constituents. Owing to the low bulk density and specific gravity of lightweight aggregates, segregation is a common phenomenon in the concrete mix where the constituents contain lightweight aggregates and natural fine sand. However, the extent can be controlled by the choice of suitable grading and by care in handing. A higher viscosity of fresh cement paste component militates against the downward movement of the heavier particles. Therefore, low water/cement ratio of the mix is less prone to segregation. Moreover, vibration provides a most valuable means of compacting concrete, but the danger of segregation is increased because a large amount of work is done on

Table 7. Relation between Rebound Number and different layers of eight samples.

\begin{tabular}{lcccccccc}
\hline Rebound number & Sample 1 & Sample 2 & Sample 3 & Sample 4 & Sample 5 & Sample 6 & Sample 7 & Sample 8 \\
\hline Top & 24.2 & 23.6 & 25.5 & 24.8 & 24.1 & 25.5 & 24.6 & 25.9 \\
Middle & 24.4 & 24.7 & 27.9 & 25.6 & 25.4 & 27.6 & 25.3 & 26.1 \\
Bottom & 26.3 & 27.1 & 28.2 & 28.9 & 28.1 & 30.1 & 29.9 & 29.4 \\
\hline
\end{tabular}


Table 8. Relation between number of surface aggregates $>5 \mathrm{~mm}$ and different layers of eight samples.

\begin{tabular}{lcccccccc}
\hline $\begin{array}{l}\text { Number of surface } \\
\text { aggregates }(>\mathbf{5 m})\end{array}$ & Sample 1 & Sample 2 & Sample 3 & Sample 4 & Sample 5 & Sample 6 & Sample 7 & Sample 8 \\
\hline Top & 78 & 79 & 76 & 76 & 75 & 69 & 71 & 74 \\
Middle & 41 & 40 & 38 & 41 & 40 & 37 & 39 & 39 \\
Bottom & 31 & 29 & 29 & 26 & 28 & 25 & 26 & 28 \\
\hline
\end{tabular}

the concrete. This is particularly slow when vibration is allowed to continue too long, thus the phenomenon of separating the coarse aggregates and the cement paste become more serious.

Bulk density is a function both of the density of the aggregate particles and the voids content between the particles. Low bulk density due to low density of aggregates means that there is large volume of space within the aggregate particles, especially the coarse aggregates. Since the pores in the coarse aggregates may not be fully interconnected, some of the pores are still filled with air after contact with water during mixing. Therefore, the relative water absorption ability is lower in the coarse aggregates than in the fine aggregates, because the relative effect of discontinuous pores is less in the fine aggregates than in the coarse aggregates. As a result, the difference in density between the coarse aggregates and the fine aggregates is increased after contact with water. The relative lower density air-filled coarse aggregates have a greater tendency to segregate when mixing.

\section{CONCLUSION}

The relationship between water permeability and different levels of the tested layers from the top surface of the lightweight concrete samples has been determined. According to the results of water permeability test by GWT, the permeability coefficient $\mathrm{K}$ of the three layers in each set of samples tends to decrease as the displacement of the tested layer from the top surface from the top surface increase.

It is the permeability of the hardened cement paste that has the greatest effect on the permeability of the concrete. However, in this experiment, all sets of sample have the same design mix proportion and preparation condition. The effect of the hardened cement on the permeability is the same. Therefore, the crucial factors influence our research on water permeability is the voids between the aggregates. So, the size of voids is crucial. Larger voids means cement paste filled and therefore more permeable of the concrete.

Segregation of the mix leading to the floatation of coarse lightweight aggregates to the top surface in the fresh state is the main factor of the cause of difference in size of the voids between aggregates and cause the different water permeability in different layers. Segregation is due to differences in the size of particles and in the specific gravity of the mix constituents and over-vibration of fresh concrete. So, excessive vibration is avoided by compacting the concrete until the surface is relatively smooth.

Larger Rebound Number and higher density in the bottom layer and more coarse lightweight aggregate in the top layer prove the existence of floatation of lightweight aggregates and explain for the descending water permeability trend. A summary of correlation between different characteristics of the layers is shown in Table 9. It can be concluded that harder and denser lightweight concrete has lower water permeability.

Table 9. Summary of correlation between different characteristics of layer.

\begin{tabular}{lccc}
\hline & Top & Middle & Bottom \\
\hline $\begin{array}{l}\text { Number of coarse } \\
\text { aggregates }\end{array}$ & More & Medium & Less \\
Density & Lower & Medium & Higher \\
Strength & Lower & Medium & Higher \\
Water permeability & Higher & Medium & Lower \\
\hline
\end{tabular}

The permeability of concrete can influence the durability when the concrete exposes to environmental attack. Segregation of the mix during handling and placing should be prevented as much as possible in order to make a consistent mix and a durable lightweight concrete. It can be achieved by lowing the water/cement ratio of the mix or using a thickening admixture since a higher viscosity of fresh cement paste component militates against the downward movement of the heavier particles. Air entrainment to remedy grading deficiencies and increase cohesiveness is also recommended. Larger-scale entrainment of air may be necessary owing to the prevalence of irregularly shaped material that is harsh and difficult to finish.

\section{REFERENCES}

BS1881:Part 110:1983. (1983). Method for making test cylinders from fresh concrete. London, England: British Standard Institution. 
BS1881:Part 111:1983. (1983). Method of normal curing of testing specimens. London, England: British Standards Institution.

BS1881:Part 116:1983. (1983). Method for determination of compressive strength of concrete cubes. London, England: British Standard Institution.

BS1881:Part 125:1986. (1986). Methods for mixing and sample fresh concrete in the laboratory. London, England: British Standard Institution.

BS1881:Part 202:1986. (1986). Recommendations for surface hardness testing by rebound hammer. London, England: British Standard Institution.

BS3797:1990. (1958). Specification and physical properties of hardened Portland cement paste. Journal of American Ceramic Society, 41, 469-504.

BS812:Part 103:1985. (1985). Method for determination of particle size distribution. London, England: British Standard Institution.

Bungey, J. H., \& Millard, S. G. (1996). Testing of concrete in structures (3rd ed.). Glasgow, Scotland: Blackie Academic \& Professional.

Concrete Society. (1988). Permeability testing of site concrete $-A$ review of methods and experience
(Technical Report 31). London, England: Concrete Society.

Federation Internationale de la Precontrainte (FIP). (1983). FIP manual of lightweight aggregate concrete (2nd ed.). London, England: Surrey University Press.

German Instruments. GWT instruction and maintenance manual.

Neville, A. M. (1995). Properties of concrete (4th ed.). Harlow, England: Longman.

Neville, A. M., \& Brooks, J. J. (1990). Concrete technology. Harlow, England: Longman.

Power, T. C. (1958). Structure and physical properties of hardened Portland cement paste. Journal of American Ceramic Society, 41, 1.

Powers, T. C., Copeland, L. E., Hayes, J. C., \& Mann, H. M. (1954). Permeability of Portland cement paste. Journal of American Concrete Institute, 51, 285-298.

Schmidt. Operating instructions for concrete test hammer types $L$ and $L R$.

Spratt, B. H. (1980). An introduction to lightweight concrete. Wexham Springs, England: Cement and Concrete Association. 